\title{
Game Theoretical Multi-Agent Modelling of Coalition Formation for Multilateral Trades
}

\author{
Chris S.K. Yeung \\ Ada S.Y. Poon \\ Felix F. Wu, Fellow, IEEE \\ Department of Electrical \& Electronic Engineering \\ University of Hong Kong \\ Hong Kong
}

\begin{abstract}
In recent years, the electric utility industries world wide have been undergoing deregulation to introduce competitiveness in the generation, transmission, and distribution of electric power. The once centralised system planning and operation management must be remodelled to adapt to the new market structure. In particular, the trading mechanism needs to be totally revised as any party may now get involved in this free-market, subject to the unavoidable constraints such as generation volumes, physical transmission means, and days-ahead scheduling. This paper presents a multi-agent model in conjunction with game theory to resolve the coalition formation for multilateral trades [1]. We have implemented the model using the Java programming language and the JATLite/JAT0.3 agent development tools developed by Stanford University.
\end{abstract}

Keywords: Game theory, multi-agent system, coalition formation, multilateral trades;

\section{INTRODUCTION}

In the past, the production and distribution of electric power was viewed as natural monopoly due to the enormous economic scale involved in the construction of such power plants and transmission systems. The franchised monopoly, insulated from competition, obviously has a much weaker incentive to control cost and avoid poor decisions than would be in an open market. As such, customers are imposed excessive costs that are economically unjustifiable.

Instead of pricing the generation, transmission, and distribution services as a bundled commodity, now these services can be offered as individual service providers. As a consequence, a far greater number of participants, with many of them previously excluded, may involve in the trading competition to assure a cost effective, market-driven electric facility to end users.

PE-432-PWRS-0-07-1998 A paper recommended and approved by the IEEE Power System Analysis, Computing and Economics Committee of the IEEE Power Engineering Society for publication in the IEEE Transactions on Power Systems. Manuscript submitted August 26, 1997; made available for printing June 22, 1998.
Following this, $\mathrm{Wu}$ and Varaiya [1] have proposed a new paradigm in which the decision mechanisms regarding the economics and reliability of system operation are separated. In their model, the economic decision should be carried out through private multilateral trades among generators and consumers whereas system security and reliability is better left to an independent system operator [2]. They have also proved that multilateral contract involving three or more parties are necessary for efficient market operation.

Based on that result, we attempt to model the trading process using multi-agent theory. Such a model addresses the problem of how market participants negotiate for multilateral contracts that are mutually beneficial without a central coordinating figure. Each agent imitates the behaviour of an electricity market participant which can be a generator, a customer, a transmission owner or any interested party.

In the next section, we will first outline a method for coalition formation in the form of agent cooperation and coordination. Since each agent is autonomous and rational by definition, it provides its own criteria to determine which agent to be partnered has a better payoff. In Section III, we will introduce the cooperative standard of fairness (C-SOF) and the Shapley value [3] often used in game theory to derive and specify these criteria. In Section IV, we will present the agent models with increasing complexity in handling and dividing transmission network cost. Finally, the computation for payoffs among agents at the end of coalition formation is given.

We have developed a multi-agent system for our model using Java and the JATLite/JAT0.3 agent tools from Stanford in order to achieve platform independence and internet accessibility.

\section{COALITION FORMATION}

The coalition formation is a process of forming a profitable partnership for a group of participants. Our method is based on Ketchpel [3] with modification to the new power market environment. Klush and Shehory also modified Ketchpel's algorithm for the cooperative recognition process within the Federative Cell System for discovery of Inter-database dependencies (FCSI) [4]. Our algorithm comprises of four phases instead of three in Ketchpel's. These four phases are described in order as followed. 


\section{A. Local information derivation phase}

Each agent first derives its local information, for example, the cost of generation or benefit of consumption of electric power, and then determines the profit when acting alone. This profit is called the agent's self-value. The self-value would then be the minimum profit that the agent could achieve without cooperating with others. The derivation of such local information depends on the agent's environment and will be discussed in Section IV.

\section{B. Communication phase}

Communication among agents allows them to locate other agents that may have beneficial trades if they collaborate. During this phase, each agent exchanges its local information derived earlier including the self-value with other agents either directly or indirectly through a coordinator. It also gathers information from an independent system operation agent concerning the surrounding environment, if any. The information exchanged between agents should be sufficient to calculate the joint payoffs.

\section{Local calculation phase}

Once each agent has the requested information from all other agents in the environment, the local calculation phase begins. Here, each agent calculates the share of benefit if teaming with another agent and creates a preference ordering with respect to the shares. The field of game theory offers several alternatives for criteria that may be useful to divide the group utility. In this paper, we adopt the cooperative standard of fairness (C-SOF) and the Shapley value to be discussed in Section III.

\section{Bilateral negotiation phase}

In this phase, each agent maintains a preference list of those agents with which it can collaborate beneficially. The agents then extend offers to each other according to their preference lists, accept offers that may improve their positions, and decline others. The newly formed coalition will behave like one decision-making unit, i.e. a new-born agent, from then on.

Notice that the four phases will be iterated in order until no more coalition is possible or a pre-determined time limit has reached.

\section{NEGOTIATION PREFERENCE LIST}

We now discuss the two approaches in establishing the negotiation preference list in the third phase of the coalition formation process.

\section{Common extra payoff}

The first approach is to use the common extra payoff as a criterion. The common extra payoff of a new coalition is the difference between the payoff of the new coalition and the sum of the payoff of its founder agents, that is, the excess paycff. In using this criterion, we have made the assumption that the founder agents in the new coalition have an equal share of the excess and this is exactly the idea of cooperative standard of fairness (C-SOF). The C-SOF assigns each negotiation group with a fair share that consists of the superadditive cover of its value plus an equal share of the excess of the maximum joint gain minus the sum of each negotiation group's maximum joint gain [8]. The superadditive cover refers to the maximum obtainable joint profit of a coalition in cooperative game theory.

Another similar and intuitive criterion is the relative weighting. Unlike the equal share of excess payoff among agents in C-SOF, a stronger agent should have a larger share of the excess. In this context, agent $A$ is stronger than agent $B$ if and only if the payoff of agent $A$ when acting alone is greater than that of agent $B$.

\section{Bilateral Shapley Value}

The second approach is introduced by Ketchpel in his proposed algorithm, the so-called Bilateral Shapley Value (BSV). It avoids the exponential complexity of Shapley value calculation. The Shapley value is calculated by looking at each of the different dynamics that could lead to the coalition under consideration.

We assumed that agents form a coalition either by being the founder or by joining one at a time with a coalition that already exists. Hence the set of formation dynamics is simply the permutations of the agents in the coalition. Each agent adds value to a given formation process of the coalition based on the marginal utility gain created by that agent. By averaging an agent's marginal contribution across all the different formation possibilities, its Shapley value is obtained. This calculation ensures that the sum of the Shapley values for all of the members of the coalition will be exactly the coalition's combined value. The calculation of Shapley value requires looking at all of the permutations and this is an exponential operation. But the computation of Shapley values for coalition of two agents is relatively small and the values are termed the Bilateral Shapley Values.

Consider a game with a set of $N$ players and characteristic function $v$, for a potential coalition of two players, $A$ and $B$, the BSV of player $A$ is given by:

$$
1 / 2 v(A)+1 / 2(v(A B)-v(B))
$$

and correspondingly, the BSV of $B$ is:

$$
1 / 2 v(B)+1 / 2(v(A B)-v(A))
$$

Indeed, in a coalition of two agents, the values based on CSOF and those on bilateral Shapley value are the same. So in this paper, we use the values based on these two different approaches interchangeably.

\section{AGENT MODELLING}

Based on the above coalition formation scheme with the game theoretical approach in establishing the negotiation preference list, we can introduce the agent modelling techniques to simulate the dialogues and negotiation among market participants.

The first model is rudimentary in which the transmission network cost is ignored. In the second model, this cost will 
be incorporated in calculating the profit of a potential coalition.

Throughout the discussion, we will refer to the 4-bus system (Figure 1) to illustrate the concepts involved.

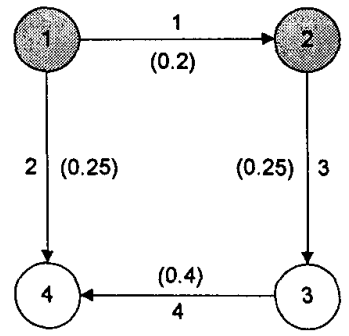

Fig. 1 A 4-bus system

In the figure, bus nodes 1 and 2 represent generators whereas bus nodes (3) and (4) represent customer loads. The number in bracket for each transmission line refers to the reactance per power unit, for example, line number 3 has a reactance of 0.25 .

\section{A. Agent model without transmission network cost}

An agent is a dispatchable process which behaves autonomously, rationally, cooperatively, and with high mobility. A multi-agent system specifies the coordination and the communication medium for agents of an application.

In our multi-agent system, an agent is naturally a generator, a customer load, or an aggregate of both. For simplicity, we do not consider fractional bus generators and loads. We also assume that any set of generation units and customer loads attached to the same bus belong to a single agent. So for the 4-bus system, we have a maximum of four agents, corresponding to the two generator buses and the two customer load buses.

We formulate the computation of joint payoff for a potential coalition $C$ as an optimization problem and find the power injection $p_{c}$ by the entities in $C$, resulting in optimal joint profit which indeed is the value of coalition $C$.

The mathematical formulation is:

$\begin{array}{ll}\text { Minimize } & \boldsymbol{u}^{T} \cdot \boldsymbol{C}\left(\boldsymbol{p}_{C}\right) \\ \text { subject to } & \boldsymbol{u}^{T} \cdot \boldsymbol{p}_{C}=0 \\ \text { Profit of coalition } C, & v(C)=-\boldsymbol{u}^{T} \cdot \boldsymbol{C}\left(\boldsymbol{p}_{C(\text { min })}\right)\end{array}$

where $C=$ vector of cost $/$ benefit functions for agents in $C$

$\boldsymbol{p}_{c}=$ vector of power injections by agents in $C$

$\boldsymbol{u}=$ unit vector, i.e., column vector of all ones

In addition, we assume that the cost of generation and the benefit of consumption, $c(q)$, vary quadratically in terms of the power injection $q$, that is:

$$
c(q)=a q+b q^{2}
$$

We now present two cases. In the first case, the costs of generation for $\mathbf{0}$ and 2 are the same, and so are the benefits of consumption for (3) and (4). In the second case, they are all different. The local information and the profit of all possible coalitions for both cases are shown in Table 1.
Local information

\begin{tabular}{|c||c|c|c|c|}
\hline \multicolumn{1}{|c||}{} & \multicolumn{2}{c|}{ Case 1 } & \multicolumn{2}{c|}{ Case 2 } \\
\cline { 2 - 5 } Bus & $a$ & $b$ & $a$ & $b$ \\
\hline \hline o & 100 & 0.05 & 100 & 0.05 \\
\hline 0 & 100 & 0.05 & 102 & 0.051 \\
\hline (3) & 120 & -0.06 & 118 & -0.059 \\
\hline (4) & 120 & -0.06 & 120 & -0.06 \\
\hline
\end{tabular}

Coalition and joint profit

\begin{tabular}{|c|c|c|c|c|}
\hline \multirow[b]{2}{*}{$\begin{array}{c}\text { Coalition, } \\
C\end{array}$} & \multicolumn{2}{|r|}{ Case 1} & \multicolumn{2}{|r|}{ Case 2} \\
\hline & Profit & $\begin{array}{c}\text { Power injection } \\
(\mathrm{MW})\end{array}$ & Profit & $\begin{array}{c}\text { Power injection } \\
(\mathrm{MW})\end{array}$ \\
\hline$\overline{\{0,(3)\}}$ & 909 & $\begin{array}{l}p_{1}=90.9091 \\
p_{3}=-90.9091\end{array}$ & 743 & $\begin{array}{l}p_{1}=82.5688 \\
p_{3}=-82.5688\end{array}$ \\
\hline$\{0,(4)\}$ & 909 & $\begin{array}{l}p_{1}=90.9091 \\
p_{4}=-90.9091\end{array}$ & 909 & $\begin{array}{l}p_{1}=90.9091 \\
p_{4}=-90.9091\end{array}$ \\
\hline$\{0,(3)\}$ & 909 & $\begin{array}{l}p_{2}=90.9091 \\
p_{3}=-90.9091\end{array}$ & 582 & $\begin{array}{l}p_{2}=72.7273 \\
p_{3}=-72.7273\end{array}$ \\
\hline$\{2,(4)\}$ & 909 & $\begin{array}{l}\mathrm{p}_{2}=90.9091 \\
\mathrm{p}_{4}=-90.9091\end{array}$ & 730 & $\begin{array}{l}p_{2}=81.0811 \\
p_{4}=-81.0811\end{array}$ \\
\hline$\{0,0,(3)\}$ & 1177 & $\begin{array}{l}\mathrm{p}_{1}=58.8235 \\
\mathrm{p}_{2}=58.8237 \\
\mathrm{p}_{3}=-117.6472\end{array}$ & 868 & $\begin{array}{l}p_{1}=60.8767 \\
p_{2}=40.0752 \\
p_{3}=-100.9519\end{array}$ \\
\hline$\{\mathbf{0}, \mathbf{2},(4)\}$ & 1177 & $\begin{array}{l}p_{1}=58.8235 \\
p_{2}=58.8237 \\
p_{4}=-117.6472\end{array}$ & 1070 & $\begin{array}{l}p_{1}=66.2021 \\
p_{2}=45.2962 \\
p_{4}=-111.4983\end{array}$ \\
\hline$\{\mathbf{0},(3),(4)\}$ & 1250 & $\begin{array}{l}p_{1}=124.9994 \\
p_{3}=-62.4997 \\
p_{4}=-62.4997\end{array}$ & 1139 & $\begin{array}{l}p_{1}=119.0727 \\
p_{3}=-51.6333 \\
p_{4}=-67.4394\end{array}$ \\
\hline$\{\mathbf{0},(3, \oplus\}$ & 1250 & $\begin{array}{l}p_{2}=124.9994 \\
p_{3}=-62.4997 \\
p_{4}=-62.4997\end{array}$ & 902 & $\begin{array}{l}\mathrm{p}_{2}=105.2139 \\
\mathrm{p}_{3}=-44.6456 \\
\mathrm{p}_{4}=-60.5682\end{array}$ \\
\hline$\{\mathbf{0}, \Theta,(1),(4)\}$ & 1818 & $\begin{array}{l}p_{1}=90.9091 \\
p_{2}=90.9091 \\
p_{3}=-90.9091 \\
p_{4}=-90.9091\end{array}$ & 1491 & $\begin{array}{l}\mathrm{p}_{1}=92.5431 \\
\mathrm{p}_{2}=71.1205 \\
\mathrm{p}_{3}=-74.1161 \\
\mathrm{p}_{4}=-89.5475\end{array}$ \\
\hline
\end{tabular}

Table 1. Two coalition formation cases - no transmission cost

It should be noted that the profit for the grand coalition $\{\mathbf{0}, \mathbf{2},(3),(4)\}$ is indeed the global optimal.

\section{A.1. Case 1 - same generation and same consumption costs}

In case 1, since the cost of generation is the same for both generators (and the benefit of consumption is the same for both consumers), there is no difference for the generators to trade with any of the customers. However, the actual path of power flow may be different. For example, the joint profits for the coalitions $\{\boldsymbol{0},(3)\}$ and $\{\boldsymbol{O},(4)\}$ are the same but the paths for the line flow are different as illustrated in Figure 2. In the figure, the outer loop represents the line flow for the coalition $\{\mathbf{0},(3)\}$ and the inner loop for that of $\{\mathbf{0},(4)\}$.

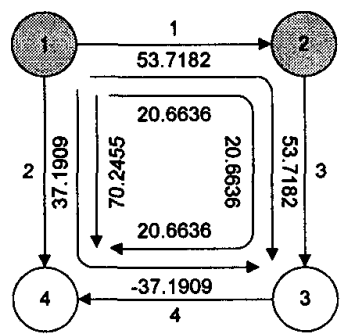

Fig. 2 Line flow for coalitions $\{0,(3)\}$ and $\{(8,4)\}-A .1$.

However, if the transmission cost is included, the profit for the above two coalitions will no longer be the same. 
Another coalition set $\{\{\boldsymbol{O},(4)\},\{\boldsymbol{( 2},(3)\}\}$ has the same total profit as the grand coalition. Again, if transmission cost is included, there will be a significant difference in the total profit as illustrated in Figure 3 and Figure 4.

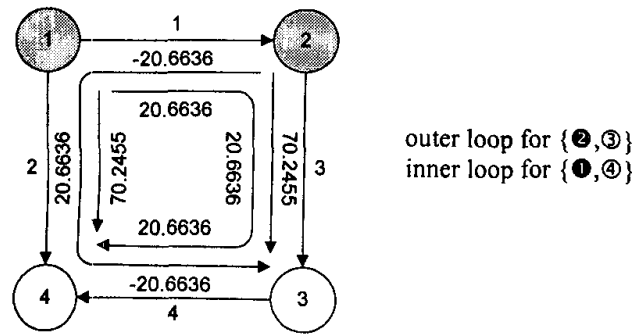

Fig. 3 Line flow for coalitions $\{0,(4)\}$ and $\{(0,3\}-A .1$.

In Figure 4, there is no power flowing along line 1 and 4 in the grand coalition. For non-negative transmission costs, the grand coalition will always have the least cost.

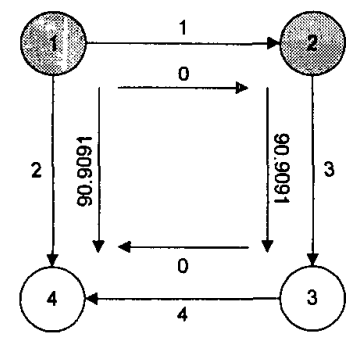

Fig. 4 Line flow for the grand coalition - A.I

\section{A.2. Case 2 - different generation and different consumption}

In case 2 , the costs of generation are different and the benefits of consumption are also different. According to Table 1 and the game theory based method in Section III, a preference list for each agent needs to be formed. The order of forming coalitions and the distribution of payoff among agents are:

$1^{\text {st }}$ round

\begin{tabular}{|c|c|c|c|c|}
\hline \multirow[b]{2}{*}{ Agent } & \multicolumn{4}{|c|}{ Preference list (from high to low) } \\
\hline & $\begin{array}{c}\text { Potential } \\
\text { agent }\end{array}$ & $\overline{\mathrm{BSV}}$ & $\begin{array}{c}\begin{array}{c}\text { Potential } \\
\text { agent }\end{array} \\
\end{array}$ & BSV \\
\hline$\{0\}$ & (6) $\}$ & 454.5 & (3) & 317.5 \\
\hline \{C\} & $\{(4)\}$ & 365.0 & (0) & 2910 \\
\hline \{3\} & $\{$ (0) & 371.5 & {$[\theta]$} & 2910 \\
\hline \{4) & (a) & 454.5 & $\{$ (2) & 365.0 \\
\hline
\end{tabular}

Coalitions formed after $1^{\text {st }}$ round are $\{\boldsymbol{0},(4)\}$ and $\{\boldsymbol{2},(3)\}$. The shaded potential agent in the table is the one to be chosen to form coalition after each round of negotiation process.

$2^{\text {nd }}$ round

\begin{tabular}{|c||c|c|}
\hline \multicolumn{1}{|c||}{ Agent } & \multicolumn{2}{c|}{ Preference list } \\
\cline { 2 - 3 } & Potential Agents & BSV \\
\hline \hline$\{\mathbf{0},(4)\}$ & $\{$ (2), (3) & 909 \\
\hline$\{$ (2, (3) & $\{\mathbf{0},(4)\}$ & 582 \\
\hline
\end{tabular}

The shared profit for $\{\mathbf{0},(4)\}$ in $\{\mathbf{0}, 2,(3),(4)\}$ is the same as when they are standalone. The same happens to $\{2,(3)\}$. So the final coalition set is $\{\{\mathbf{0},(4),\{\boldsymbol{2},(3)\}\}$. The idea behind is simply the least cost matching with the highest benefit and the process continues.

\section{B. Agent model with linear transmission network cost}

In this model, the set of autonomous agents and the assumptions are pretty much the same as before, with one exception: that the cost of using a transmission line varies linearly with its line flow (Figure 5) and the transmission network has infinite capacity.

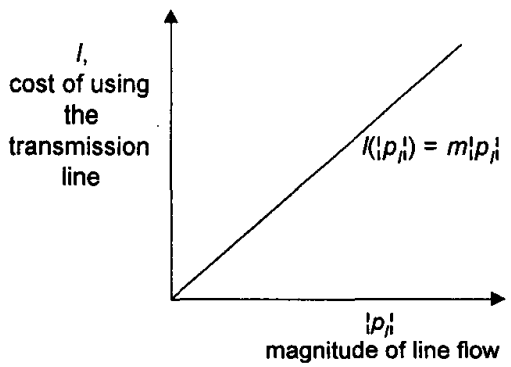

Fig. 5 Line cost versus line flow in model 2

With the transmission cost in mind, we have a new formulation for the optimization problem.

The mathematical formulation is:

Minimize $\quad u^{T} \cdot C\left(p_{C}\right)+l^{T} \cdot|H \cdot p|$

subject to

$$
\boldsymbol{u}^{T} \cdot \boldsymbol{p}_{C}=0
$$

Profit of coalition $C$,

$$
v(C)=-u^{T} \cdot C\left(p_{C(m i n)}\right)-l^{T} \cdot\left|\boldsymbol{H} \cdot p_{(m i n)}\right|
$$

where $C=$ vector of cost / benefit functions for agents in $C$

$p_{C}=$ vector of power injections by agents in $C$

$\boldsymbol{l}=$ vector of all transmission line costs

$\boldsymbol{H}=$ transfer admittance matrix of the transmission network

$\boldsymbol{p}=$ vector of all net power injections at all buses by the agents in $C$ $\boldsymbol{u}=$ column vector of all ones

We will also consider two cases here. In the first case, the transmission costs of all lines are the same as they have the same slope $m$. In the second case, the cost of line 2 increases sharply while keeping the rest unchanged. The global and local information, and the joint profit of all possible coalitions for both cases, are shown below (Table 2). Notice that the generation costs and the consumption costs are identical respectively.

Global information
\begin{tabular}{|c|c|c|}
\hline & Case 1 & Case 2 \\
\cline { 2 - 3 } Line & $m$ & $m$ \\
\hline 1 & 5 & 5 \\
\hline 2 & 5 & 13 \\
\hline 3 & 5 & 5 \\
\hline 4 & 5 & 5 \\
\hline
\end{tabular}




\begin{tabular}{|c|c|c|c|c|c|c|}
\hline \multirow{3}{*}{\begin{tabular}{c|} 
Coalition \\
$C$ \\
\end{tabular}} & \multirow{2}{*}{\multicolumn{3}{|c|}{ Case 1 }} & \multirow{2}{*}{\multicolumn{3}{|c|}{ Case 2}} \\
\hline & & & & & & \\
\hline & Profit & $\begin{array}{c}\text { Power } \\
\text { Injection }\end{array}$ & $\begin{array}{c}\text { Trans. } \\
\text { cost } \\
\end{array}$ & Profit & $\begin{array}{c}\text { Power } \\
\text { Injection }\end{array}$ & $\begin{array}{c}\text { Trans. } \\
\text { cost }\end{array}$ \\
\hline$\{\mathbf{0},(3)\}$ & 227 & $\begin{array}{l}p_{1}=45.4545 \\
p_{3}=-45.4545\end{array}$ & 455 & 103 & $\begin{array}{l}\mathrm{p}_{1}=30.5782 \\
\mathrm{p}_{3}=-30.5782\end{array}$ & 406 \\
\hline$\{\mathbf{0},(4)\}$ & 368 & $\begin{array}{l}p_{1}=57.8500 \\
p_{4}=-57.8500\end{array}$ & 421 & 97 & $\begin{array}{l}p_{1}=29.7518 \\
p_{4}=-29.7518\end{array}$ & 400 \\
\hline$\{2,(3)\}$ & 368 & $\begin{array}{l}p_{2}=57.8500 \\
p_{3}=-57.8500\end{array}$ & 421 & 270 & $\begin{array}{l}p_{2}=49.5845 \\
p_{3}=-49.5845\end{array}$ & 451 \\
\hline$\{2,4\}$ & 227 & $\begin{array}{l}p_{2}=45.4545 \\
p_{4}=-45.4545\end{array}$ & 455 & 63 & $\begin{array}{l}p_{2}=23.9673 \\
p_{4}=-23.9673\end{array}$ & 353 \\
\hline$\{\mathbf{0}, \mathbf{2},(3)\}$ & 515 & $\begin{array}{l}p_{1}=21.0012 \\
p_{2}=54.5957 \\
p_{3}=-75.5969\end{array}$ & 483 & 361 & $\begin{array}{l}p_{1}=17.5889 \\
p_{2}=45.7249 \\
p_{3}=-63.3138\end{array}$ & 545 \\
\hline$\{0,2,(4)\}$ & 515 & $\begin{array}{l}p_{1}=54.5957 \\
p_{2}=21.0012 \\
p_{4}=-75.5969\end{array}$ & 483 & 171 & $\begin{array}{l}\mathrm{p}_{1}=31.4223 \\
\mathrm{p}_{2}=12.0871 \\
\mathrm{p}_{4}=-43.5095\end{array}$ & 529 \\
\hline$\{0,(3,4)\}$ & 529 & $\begin{array}{l}\mathrm{p}_{1}=80.1359 \\
\mathrm{p}_{3}=-28.6218 \\
\mathrm{p}_{4}=-51.5142\end{array}$ & 544 & 198 & $\begin{array}{l}p_{1}=48.9450 \\
p_{3}=-17.4815 \\
p_{4}=-31.4636\end{array}$ & 584 \\
\hline$\{2,(3),(4)\}$ & 529 & $\begin{array}{l}p_{2}=80.1359 \\
p_{3}=-51.5142 \\
p_{4}=-28.6218\end{array}$ & 544 & 325 & $\begin{array}{l}\mathrm{p}_{2}=62.8105 \\
\mathrm{p}_{3}=-40.3768 \\
\mathrm{p}_{4}=-22.4337\end{array}$ & 606 \\
\hline$\{0,2,(3),(4)\}$ & 1023 & $\begin{array}{l}p_{1}=68.1821 \\
p_{2}=68.1821 \\
p_{3}=-68.1821 \\
p_{4}=-68.1821\end{array}$ & 682 & 551 & $\begin{array}{l}\mathrm{p}_{1}=48.7643 \\
\mathrm{p}_{2}=51.2357 \\
\mathrm{p}_{3}=-52.7807 \\
\mathrm{p}_{4}=-47.2193\end{array}$ & 898 \\
\hline
\end{tabular}

Table 2. Two coalition formation cases - linear transmission cost

\section{B.1. Case 1 - same transmission line cost}

In case 1 , the transmission costs for all lines are the same. The coalition formation process is illustrated below:

\section{$\underline{1^{\text {st }} \text { round }}$}

\begin{tabular}{|c|c|c|c|c|}
\hline \multirow[b]{2}{*}{ Agent } & \multicolumn{4}{|c|}{ Preference list } \\
\hline & $\begin{array}{c}\text { Potential } \\
\text { agent }\end{array}$ & BSV & $\begin{array}{c}\text { Potential } \\
\text { agent }\end{array}$ & BSV \\
\hline$\{0\}$ & (ब1) & 1840 & (3) & $\bar{~} 1113.5$ \\
\hline$\{0\}$ & (O) & 184.0 & (4) & 113.5 \\
\hline$\{(3)\}$ & $(2)$ & 184.0 & $\{0\}$ & 113.5 \\
\hline$\{(4)\}$ & D. & 184.0 & $\{2\}$ & 113.5 \\
\hline
\end{tabular}

The coalitions formed after the $1^{\text {st }}$ round are $\{\boldsymbol{O},(4)\}$ and $\{(3,3)$. The payoff configuration (PC) is thus $(184,184$, $184,184 ;\{\mathbf{1},(4)\},\{2,(3)\})$.

$2^{\text {nd }}$ round

\begin{tabular}{|c||c|c|}
\hline \multicolumn{1}{|c||}{ Agents } & \multicolumn{2}{c|}{ Preference list } \\
\cline { 2 - 3 } & Potential Agents & BSV \\
\hline \hline$\{\mathbf{0},(4)\}$ & $\{\mathbf{Q}$, (3) $\}$ & 511.5 \\
\hline$\{\mathbf{Q}$, (3) $\}$ & $\{\mathbf{0},(4)\}$ & 511.5 \\
\hline
\end{tabular}

After the $2^{\text {nd }}$ round, the grand coalition is formed and the corresponding PC is $(255.75,255.75,255.75,255.75$; $\{\mathbf{0}, \mathbf{2},(3$, (4) $\}$ ).

Now, we investigate the line flow for the coalition $\{\boldsymbol{O},(3)\}$ and $\{\boldsymbol{O},(4)\}$ as depicted in Figure 6 and compare it with the Figure 3 earlier. For coalition $\{\boldsymbol{0},(4)\}$, a larger amount of power is flowing along line 2 , which connects bus $\mathbf{O}$ and bus (4). For coalition $\{\mathbf{0},(3)\}$, there is no direct path from bus $\mathbf{0}$ to bus (3). So it is sensible that coalition $\{\boldsymbol{0},(4)$ has a higher profit than coalition $\{\boldsymbol{0}$, (3) $\}$.

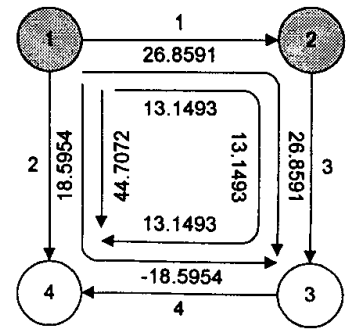

Fig. 6 Line flow for coalitions $\{0,(3)\}$ and $\{0,(4)\}-$ B.1.

When the coalitions $\{\boldsymbol{O},(4)\}$ and $\{\boldsymbol{2},(3)\}$ cooperate, the line flows along line 1 (and line 4) are counter-balanced as illustrated in Figure 4 and Figure 7 . So the cost will certainly be less than that when $\{\boldsymbol{0},(4)\}$ and $\{\boldsymbol{2},(3)\}$ work separately.

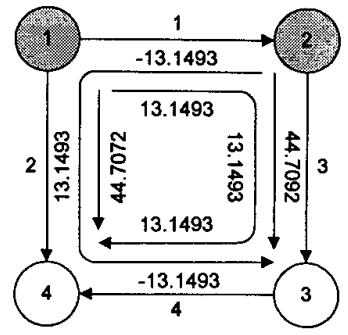

Fig. 7 Line flow for $\{\{\boldsymbol{O},(4),\{(\theta,(3)\}\}-B .1$.

In Figure 8 , there is no power flowing along line 1 and 4 in the grand coalition so its transmission cost is the least.

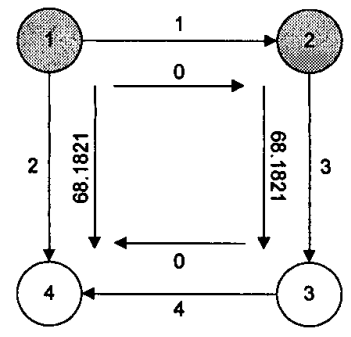

Fig. 8 Line flow for the grand coalition - B.1.

\section{B.2. Case 2 - different transmission line cost}

In case 2 where the transmission line costs are different, the coalition formation process is as follows:

$\underline{1^{\text {st }} \text { round }}$

\begin{tabular}{|c|c|c|c|c|}
\hline \multirow[b]{2}{*}{ Agent } & \multicolumn{4}{|c|}{ Preference list } \\
\hline & $\begin{array}{c}\text { Potential } \\
\text { agent }\end{array}$ & BSV & $\begin{array}{c}\begin{array}{c}\text { Potential } \\
\text { agent }\end{array} \\
\end{array}$ & BSV \\
\hline$\{0\}$ & $\{3\}$ & 51.5 & (ब) & 48.5 \\
\hline$\{2\}$ & (1) & 1350 & $\{$ (4) $\}$ & 31.5 \\
\hline$\{(3)\}$ & (2) & 135.0 & $\{\mathbf{0}\}$ & 51.5 \\
\hline$\{(4)\}$ & $=(\mathbf{0})$ & 48.5 & $\{0\}$ & 31.5 \\
\hline
\end{tabular}

Agent $\{(0\}$ extends offer to $\{3\}$ and at the same time, $\{(0\}$ extends offer to $\{3\}$. Since in the preference list of $\{(3),\{2\}$ is at a higher position than $\{0\}$, so $\{3\}$ accepts the offer from $\{2\}$ and declines that from $\{\boldsymbol{O}\}$. Being rejected by $\{3\},\{\mathbf{O}\}$ extends offer to $\{4\}$ which is next to $\{(3)$ in its preference. $\{(4\}$ accepts the offer from $\{\mathbf{0}\}$. So 
coalitions formed after the $1^{\text {st }}$ round are $\{\boldsymbol{( 2 ,}$, (3) $\}$ and $\{\boldsymbol{0},(4)\}$. The $\mathrm{PC}$ is $(48.5,135,135,48.5 ;\{\boldsymbol{\theta},(3)\},\{\boldsymbol{0},(4)\})$.

$\underline{2}^{\text {nd }}$ round

\begin{tabular}{|c||c|c|}
\hline \multirow{2}{*}{ ?Players } & \multicolumn{2}{c|}{ Preference list } \\
\cline { 2 - 3 } & Potential Players & BSV \\
\hline \hline$\{\mathbf{0},(4)\}$ & $\{(2,(3)$ & 189 \\
\hline$\{(2$, (3) & $\{\mathbf{0},(4)\}$ & 362 \\
\hline
\end{tabular}

Coalition formed after the $2^{\text {nd }}$ round is the grand coalition $\{1,2,3,4\}$. The PC is $(94.5,181,181,94.5 ;\{1,2,3,4\})$.

Now, the cost for one of the lines, line 2, is extraordinary high relative to the other three. Due to the increased cost in transmission, the profit for each coalition drops in comparison with that of case 1 . Here, despite the existence of direct path between bus $\mathbf{0}$ and bus (4), the profit for coalition $\{\boldsymbol{0},(4)\}$ is less than that for coalition $\{\mathbf{0},(3)\}$ as depicted in Figure 9.

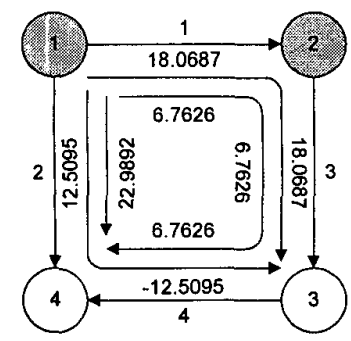

Fig. 9 Line flow for coalitions $\{0,(3)\}$ and $\{0,(4)\}-B .2$.

\section{PROFIT ALLOCATION}

So far, we only consider the formation of coalition strictures dynamically. We have not discussed the allocation of payoffs among the agents. Once the coalition structure is known, there are many solution concepts to derive the payoff vectors, for example, the core, stable set and Shapley value from game theory. But in order to be consistent with the evolution of the coalition structure, the allocation method should be consistent with the criterion in establishing the neg,otiation preference list. Otherwise, the coalition structure so formed will not be stable. For example, if we base on a "fair" partition of the excess payoff in establishing the preference list, then in profit allocation, we should also follow the same partition approach.

Now consider a game of 4 agents named $A, B, C$ and $D$. We assume that the criterion in establishing the negotiation preference list is based on the cooperative standard of faimess (C-SOF). Then the coalition formation stages are: $\{\{A\},\{B\},\{C\},\{D\}\} \rightarrow\{\{A, B\},\{C, D\}\} \rightarrow\{A, B, C, D\}$. According to proposed allocation method, the profit for $\{A, B\}$ in $\{A, B, C, D\}$ will be its value plus an equal share of the difference between the joint profit, and the sum of its value and its partner's value, $P_{A B}$ say. That is

$$
P_{A B}=v(A B)+\frac{1}{2}(v(A B C D)-v(A B)-v(C D))
$$

Similarly,

$$
P_{C D}=v(C D)+\frac{1}{2}(v(A B C D)-v(A B)-v(C D))
$$

The profit for $A, P_{A}$ will be

$$
P_{A}=v(A)+\frac{1}{2}\left(P_{A B}-v(A)-v(B)\right)
$$

and the profit for all the other agents are calculated similarly.

\section{CONCLUSION AND FUTURE WORK}

In this paper, we have introduced a new idea in developing multilateral trades using game theory and multi-agent modelling. The objective is to derive a workable and profitable coalition partnership under the fair play practice.

The agent models discussed in this paper are relatively simple to the extent that bilateral trade seems suffice. However, the variation in transmission line costs, inclusion of counter-flow within a coalition, and release of transmission constraints by having more parties involved in a trade contributes to the preference of multilateral trade. We are in the process of defining more models with higher order of complexity such as including the transmission network constraints to secure power generation and transmission conditions. To the far end, we attempt to model the transmission network owners as agents instead of acting purely as service providers.

\section{REFERENCES}

[1] F.F. Wu and P. Varaiya, "Coordinated Multilateral Trades for Electric Power Networks: Theory and Implementation", Program on Workable Energy Regulation PWP-03I, June 1995.

[2] "Independent System Operator [ISO] and Power Exchange [PX] Communications Infrastructure Systems and Services Design and Acquisition Project Request for Proposal [RFP]", California, U.S. August 23, 1996

[3] S.P. Keptchpel, "Coalition Formation Among Autonomous Agents", Proceedings of MAAMAW-93, Springer-Verlag, 1993.

[4] M. Klush and O. Shehory, "Coalition Formation Among Rational Information Agents", Proceedings of MAAMAW-96, Springer-Verlag, 1996.

[5] F. von Martial, "Coordinating Plans of Autonomous Agents", Lecture Notes in Artificial Intelligence, vol. 610, Springer-Verlag, Berlin, Germany, 1992.

[6] M. Wooldridge and N.R. Jennings, "Intelligent agents: Theory and practice", The Knowledge Engineering Review, 1995

[7] M. Barbuceanu and M.S. Fox, "Capturing and Modeling Coordination Knowledge for Multi-Agent System", International Journal of Intelligent and Cooperative Information Systems, 1996

[8] J.P. Kahan and A. Rapoport, A."Theories of Coalition Formation", Lawrence Erlbaum Associates, 1984.

\section{BIOGRAPHIES}

Chris S.K. Yeung has been a lecturer with the Department of Electrical \& Electronic Engineering of the University of Hong Kong since 1987. He received his B.Sc. degree in Mathematics from Simon Fraser University and a M.Math degree in Computer Science from University of Waterloo. His research areas include internet technologies and VLSI systems

Ada S.Y. Poon is an M.Phil candidate in the Department of Electrical \& Electronic Engineering of the University of Hong Kong. She graduated with a first class B.Eng. degree in 1996 from the same University. She will be working towards her Ph.D. at U.C. Berkeley starting September 1997.

Felix F. Wu joined the University of Hong Kong as the chair professor of Electrical Engineering in September 1995. Later in May 1997, he has been appointed as the pro vice-chancellor of the University. Prior to that, he was a professor and the vice chairman of the Department of EECS, U.C. Berkeley, the same institute receiving his $\mathrm{Ph} . \mathrm{D}$. degree. His research interests cover a wide range of topics in power system planning and operation, including economics and reliability in system planning, real-time security assessment, and design of energy management systems and distribution automation. Recently he has been involved in the design of industry restructuring and electricity pricing in California and elsewhere. 